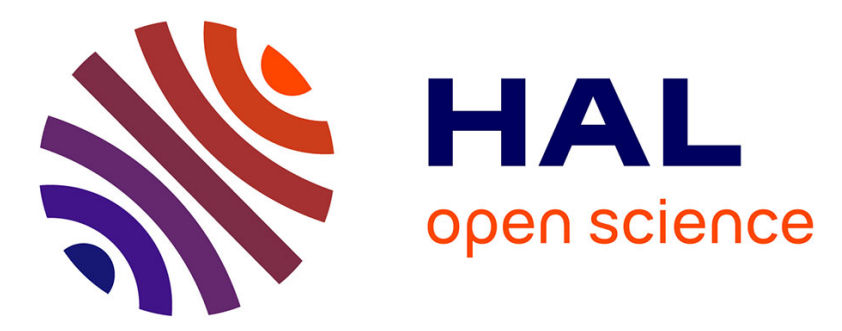

\title{
Evapotranspiration en zone semi-aride de deux couverts végétaux (gazon, blé) obtenue par plusieurs méthodes. II. - Méthodes neutroniques et tensiométriques
}

Georges Vachaud, Michel Vauclin, Charles Riou, Zouhaier Chaabouni

\section{- To cite this version:}

Georges Vachaud, Michel Vauclin, Charles Riou, Zouhaier Chaabouni. Evapotranspiration en zone semi-aride de deux couverts végétaux (gazon, blé) obtenue par plusieurs méthodes. II. - Méthodes neutroniques et tensiométriques. Agronomie, 1985, 5 (3), pp.267-274. hal-00884758

\section{HAL Id: hal-00884758 \\ https://hal.science/hal-00884758}

Submitted on 1 Jan 1985

HAL is a multi-disciplinary open access archive for the deposit and dissemination of scientific research documents, whether they are published or not. The documents may come from teaching and research institutions in France or abroad, or from public or private research centers.
L'archive ouverte pluridisciplinaire HAL, est destinée au dépôt et à la diffusion de documents scientifiques de niveau recherche, publiés ou non, émanant des établissements d'enseignement et de recherche français ou étrangers, des laboratoires publics ou privés. 


\title{
Evapotranspiration en zone semi-aride de deux couverts végétaux (gazon, blé) obtenue par plu- sieurs méthodes. II. - Méthodes neutroniques et tensiométriques
}

\author{
Georges VACHAUD, Michel VAUCLIN, Charles RIOU*, Zouhaier CHAABOUNI** \\ C.N.R.S., Institut de Mécanique de Grenoble, B.P. 68, F 38402 Saint-Martin-d'Heres Cedex \\ * I.N.R.A., Station de Bioclimatologie - Télédétection, route de Saint-Cyr, F 78000 Versailles \\ ** Centre de Recherches du Génie Rural, Ministère de l'Agriculture, DERV, B.P. 10, Tunis-Ariana-Tunisie
}

On présente les résultats d'évaluations de l'évapotranspiration réelle d'une culture pluviale de blé (sur $10000 \mathrm{~m}^{2}$ ) et d'un gazon irrigué (sur $4000 \mathrm{~m}^{2}$ ) effectuées près de Tunis pendant 2 années consécutives, par l'utilisation simultanée d'un humidimètre à neutrons et de tensiomètres.

L'implantation des sites de mesures (un site pour le gazon ; 4 sites pour le blé) a donné lieu à une étude spécifique afin de juger leur représentativité spatiale.

Les évapotranspirations observées par période moyenne de 2 semaines sont comparées aux estimations par la formule de Penman modifiée selon Riou \& CharTier (1 ${ }^{\text {re }}$ partie de cet article).

On montre que si l'on prend soin de sélectionner les sites de mesures pour leur représentativité spatiale, les mesures d'évapotranspiration sont tout à fait comparables aux estimations d'évapotranspiration potentielle tant que la réserve hydrique du sol n'est pas limitante. Ces mesures confirment que durant cette phase, la consommation du blé est supérieure à celle du gazon, comme le suggèrent les résultats présentés par RIOU \& CHARTIER.

Il est également mis en évidence que la méthode du bilan hydrique pose problème pour évaluer l'évapotranspiration lors de pluies intenses ou d'irrigations non uniformes conduisant à du ruissellement. Dans ce cas, l'estimation climatologique de l'évapotranspiration potentielle, couplée aux mesures du bilan hydrique dans le sol, permet d'estimer le ruissellement.

Mots clés additionnels : Evapotranspiration potentielle, formule de PENMAN, variabilité spatiale, bilan hydrique du sol.

Use of different methods to estimate evapotranspiration from two plant covers (grass and wheat) in the semi-arid zone. II. - Soil moisture measurement with neutron probe and tensiometers.

Water balance under two crops (rain-fed wheat and irrigated grass) was evaluated during two consecutive years in an experimental field near Tunis by the use of a neutron probe and tensiometers. Special care was given to the selection of spatially representative sites of measurement. Values of actual evapotranspiration measurements every two weeks were compared with estimates of potential evapotranspiration obtained by the PENMAN formula as modified by RIOU \& CHARTIER (first part of this paper). If measurement sites properly selected for their spatial representativity, soil measurements of evapotranspiration agree very well with potential estimations so long as soil water is not a limiting factor. During this period, water consumption by wheat was higher than by grass as suggested by the climatological results obtained by RIOU \& CHARTIER. The soil water balance method was highly questionable for estimating evapotranspiration rates in the case of heavy rainfall leading to runoff. In this case, climatological determination of potential evapotranspiration coupled with water balance measurements in the soil could be used to estimate runoff.

Additional key words : Potential evapotranspiration, PENMAN's formula, spatial variability, soil water balance. 


\section{INTRODUCTION}

L'estimation de l'évapotranspiration réelle (ETR) d'un couvert végétal par l'utilisation simultanée d'un humidimètre neutronique et de tensiomètres a fait l'objet de nombreuses études au cours de ces dernières années (DANCETTE et al., 1979 ; KALMS et al., 1982). A l'inverse des méthodes de type microclimatologique, fondées sur la mesure du bilan d'énergie et des transferts de quantité de mouvement dans la couche limite de surface (PERRIER et al., 1980), les mesures effectuées dans le sol présentent l'inconvénient très important de n'être que ponctuelles. L'aptitude de cette méthode à déterminer le comportement hydrique d'une parcelle se heurte à la variabilité naturelle du sol et de la culture et elle est conditionnée à l'implantation de plusieurs sites de mesures.

On présente ici les résultats relatifs aux consommations en eau (ETR) d'une culture pluviale de blé et d'un gazon irrigué déterminées en des points sélectionnés (VACHAUD et al., 1983) selon des critères d'indépendance spatiale et de représentativité de valeurs caractéristiques de la fonction de répartition des variables d'intérêt (agents texturaux, humidité).

L'expérimentation est réalisée sur un site installé dans la plaine du Mornag $(20 \mathrm{~km}$ au Sud-Est de Tunis).

Dans la $1^{\text {re }}$ partie de cet article, RIOU \& CHARTIER (1985) comparent sur le même site différentes méthodes (évapotranspiromètre, bacs d'évaporation : Colorado enterré et classe $A$, formule de PENMAN) d'estimation de l'évapotranspiration en conditions hydriques optimales (ETP). Ces auteurs montrent l'intérêt du bac d'évaporation et notamment du bac enterré pour estimer l'ETP gazon. De plus, la formule de PENMAN adaptée à la Tunisie rend bien compte de l'évapotranspiration de l'eau libre et de l'ETP gazon, par comparaison aux mesures en bac et en évapotranspiromètre.

Dans le présent article, ces résultats sont confrontés avec ceux obtenus par la méthode du bilan hydrique dans le but :

- de comparer les données d'ETR déterminées par différentes méthodes,

- d'estimer les déficits hydriques en condition d'alimentation hydrique insuffisante.

\section{MÉTHODES ET TECHNIQUES}

L'étude effectuée pendant 2 années (octobre 1980octobre 1982) porte sur les 2 parcelles de la ferme expérimentale de l'Institut National de Recherches Agronomiques de Tunisie située dans la plaine du Mornag. La $1^{\text {re }}$ parcelle de $5000 \mathrm{~m}^{2}$ localisée à l'intérieur de la station hydrométéorologique aménagée par l'ORSTOM (dans le cadre d'une collaboration avec le Ministère de l'Agriculture de Tunisie et la Direction des ressources en eau et en sol) est couverte en gazon (kikuyu) irrigué en période sèche. La $2^{\mathrm{e}}$ parcelle de $10000 \mathrm{~m}^{2}$, adjacente à la station est comprise dans une zone de 2 ha cultivés en blé sans irrigation. La description de la station et les conditions climatiques sont données en détail par RIOU \& CHARTIER (1985).
Le sol est un dépôt alluvionnaire sur un ancien lit fluvial. Il présente une grande hétérogénéité sur l'épaisseur étudiée $(0-2 \mathrm{~m})$. On rencontre globalement une stratification sur 2 niveaux :

- une couche argilo-sableuse en surface dont l'épaisseur varie de 0,40 à $1,0 \mathrm{~m}$,

- une couche plus sableuse en profondeur, avec parfois présence de lentilles argileuses.

Pour les 2 traitements étudiés on dispose en tout de 6 sites de mesures représentés figure 1. Chaque site se compose d'un tube d'accès pour humidimètre neutronique jusqu'à la cote $\mathrm{z}=1,70 \mathrm{~m}$ (référence $\mathrm{TCi}$ ou TKi selon leur implantation sur la parcelle Culture (C) et Kikuyu $(K)$ et de tensiomètres connectés à des manomètres multiples à mercure).

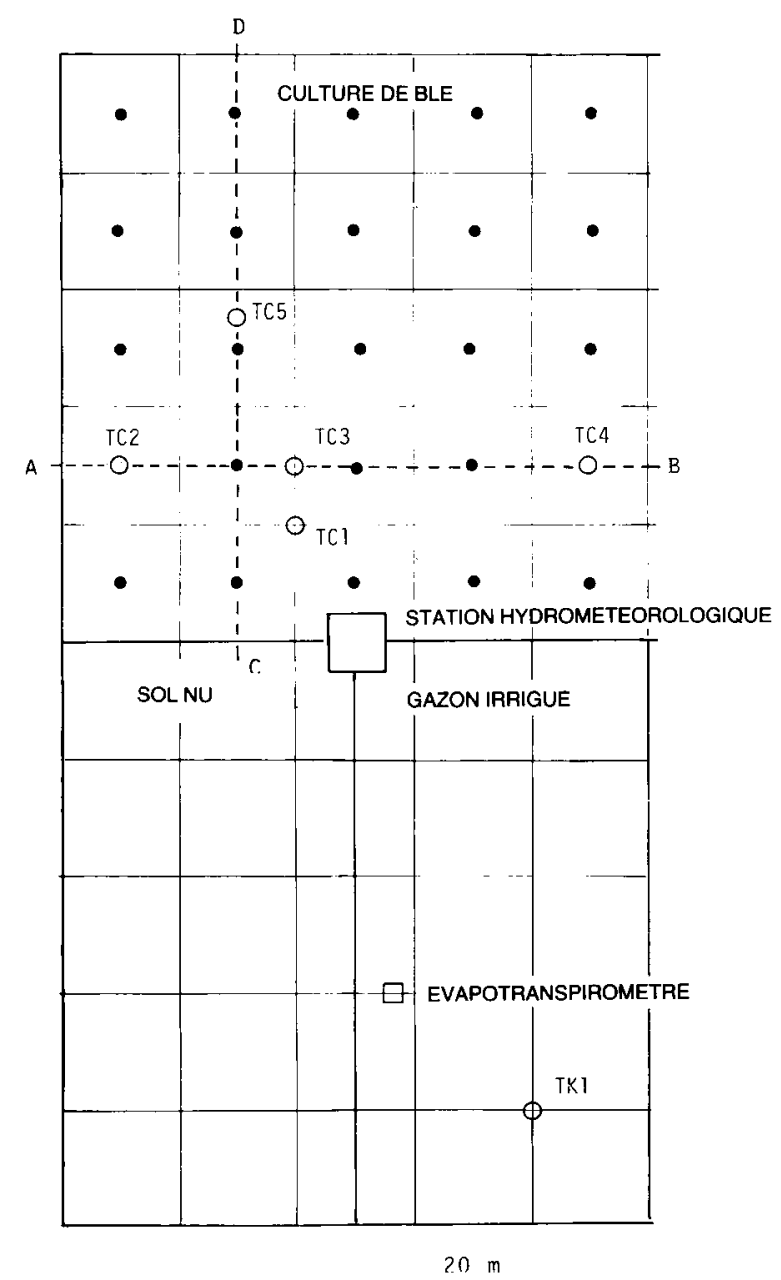

Figure 1

Schéma de l'expérimentation .

O Site de mesures (tube neutronique + tensiomètres)

- Prélèvements gravimétriques (août 1981; février, avril, juin 1982)

-- Prélèvements gravimétriques (novembre 1981) tous les $5 \mathrm{~m}$.

Experimental set-up :

O Measurement site (neutron access tube + tensiometers)

- Gravimetric samples (August 1981; February, April, June 1982)

--. Gravimetric samples (November 1981) every $5 \mathrm{~m}$.

L'humidimètre à neutrons utilisé (SOLO 20) a fait l'objet d'un étalonnage spécifique, reposant sur l'utilisation simultanée d'une sonde gamma-neutron de profondeur (CPN 502) et le prélèvement d'échantillons de sol remaniés lors de la mise en place des tubes d'accès, 
ou au voisinage immédiat de ces tubes. Le traitement statistique a été effectué selon la procédure décrite par HAVERKAMP et al. (1984).

Le site TK 1 , équipé de 10 tensiomètres $(0,15 ; 0,25$; 0,$35 ; 0,45 ; 0,55 ; 0,75 ; 0,95 ; 1,15 ; 1,45$ et $1,75 \mathrm{~m}$ ) est situé près de l'évapotranspiromètre-gazon (RIOU \& CHARTIER, 1985). Le site TCl équipé de 7 tensiomètres $(0,15 ; 0,25 ; 0,40 ; 0,55 ; 0,75 ; 1,05$ et $1,45 \mathrm{~m})$ a fait l'objet en novembre 1981 d'un essai de caractérisation hydrodynamique par la méthode du drainage interne. De ce fait, il n'a pas été utilisé pendant la campagne 1982 pour le calcul des bilans hydriques.

Les sites TC2, TC3, TC4 et TC5 sont équipés de tensiomètres aux cotes 0,$30 ; 0,75 ; 0,90$ et $1,30 \mathrm{~m}$. Leur implantation, contrairement aux sites TC1 et TK1 résulte d'une étude de la distribution spatiale de l'humidité pondérale effectuée par prélèvement d'échantillons de sol à la tarière jusqu'à $1,40 \mathrm{~m}$ de profondeur puis tous les $5 \mathrm{~m}$ le long de 2 axes orthogonaux (fig. 1). Les résultats obtenus, dont le détail est donné par VACHAUD et al. (1983) montrent l'absence d'autocorrélation entre des mesures d'humidité distantes de plus de $20 \mathrm{~m}$ et permettent d'assigner aux sites de mesures la représentativité spatiale suivante : TC3 caractérise la valeur moyenne du stock d'eau; TC4 la plus forte valeur ; TC2 et TC5 représentent le stock à environ un écart-type de la moyenne. De plus, 4 répétitions de prélèvements gravimétriques réalisés en conditions hydriques très contrastées (août 1981; février, avril et juin 1982) selon un quadrillage de $20 \times 20 \mathrm{~m}$ (fig. 1) ont permis de vérifier la stabilité temporelle de cette représentativité spatiale (VACHAUD et al., 1984) et de montrer que cette classification s'applique également pour les variations d'humidité.

Une série de prélèvements gravimétriques effectués en 8 points de la parcelle de gazon a montré que la mesure du stock hydrique obtenue en TK1 est contenue dans la bande de certitude de la valeur moyenne correspondant aux 8 observations.

En chaque site, le bilan hydrique est calculé par l'équation de conservation de la masse :

$$
\Delta \mathrm{S}=\mathrm{P}+\mathrm{I}-\mathrm{ETR}-\mathrm{D}-\mathrm{R}
$$

où $\Delta S$ est la variation du stock hydrique mesurée sur tout le profil entre 2 dates ; $P$ la pluie ; I l'irrigation ; ETR l'évapotranspiration réelle ; D le drainage ou la remontée capillaire à la base du profil et $\mathbf{R}$ le ruissellement. Toutes ces grandeurs, exprimées en $\mathrm{mm}$ d'eau, représentent les lames cumulées pendant une période de temps $\Delta \mathrm{t}$ (généralement 2 semaines) séparant les observations neutroniques. Sur le site TK1, l'analyse de toutes les mesures obtenues depuis 1980, selon la méthode décrite par KALMS et al. (1982), a conduit à estimer la relation conductivité hydraulique-teneur en eau à la cote $\mathrm{z}=1,60 \mathrm{~m}$ par l'expression :

$$
\mathrm{K}(\mathrm{mm} / \mathrm{j})=7,7 \times 10^{5} \times \theta^{6,1}
$$

où $\theta$ est la teneur en eau volumique du sol $\left(\mathrm{m}^{3} / \mathrm{m}^{3}\right)$.

L'application de la loi de Darcy généralisée permet donc d'estimer la lame d'eau écoulée à la base de ce profil par :

$$
\mathrm{D}(\mathrm{mm})=-\mathrm{K}(\theta) \cdot \frac{\mathrm{dH}}{\mathrm{dz}} \cdot \Delta \mathrm{t}
$$

où $\mathrm{dH} / \mathrm{dZ}$ est le gradient de charge hydraulique mesuré à la cote considérée.
$\mathrm{D}$ étant ainsi déterminé, l'équation (1) ne comporte plus que les inconnues ETR et R. Elle permet de calculer ETR en période sèche sachant que $R$ est alors nul. En période humide, une hypothèse supplémentaire sur l'un des 2 termes (ETR ou R) devra être faite pour calculer l'autre.

\section{RÉSULTATS}

\section{A. Estimation du bilan hydrique sous gazon irrigué}

Les différentes composantes du bilan hydrique calculées par les expressions (1), (2) et (3) sont reportées tableau 1 pour le site TK1. Les valeurs cumulées correspondantes sont données figure 2 . Les commentaires suivants peuvent être faits :

1 - Le fort ruissellement (126 mm, cf. fig. 2A) induit par les grosses pluies de décembre 1980 s'explique par 2 facteurs :

- la parcelle a été intensivement irriguée durant l'automne afin de permettre le démarrage du gazon. Cela a contribué à réduire fortement l'infiltrabilité du sol ;

- la couverture du sol par la végétation est relativement faible.
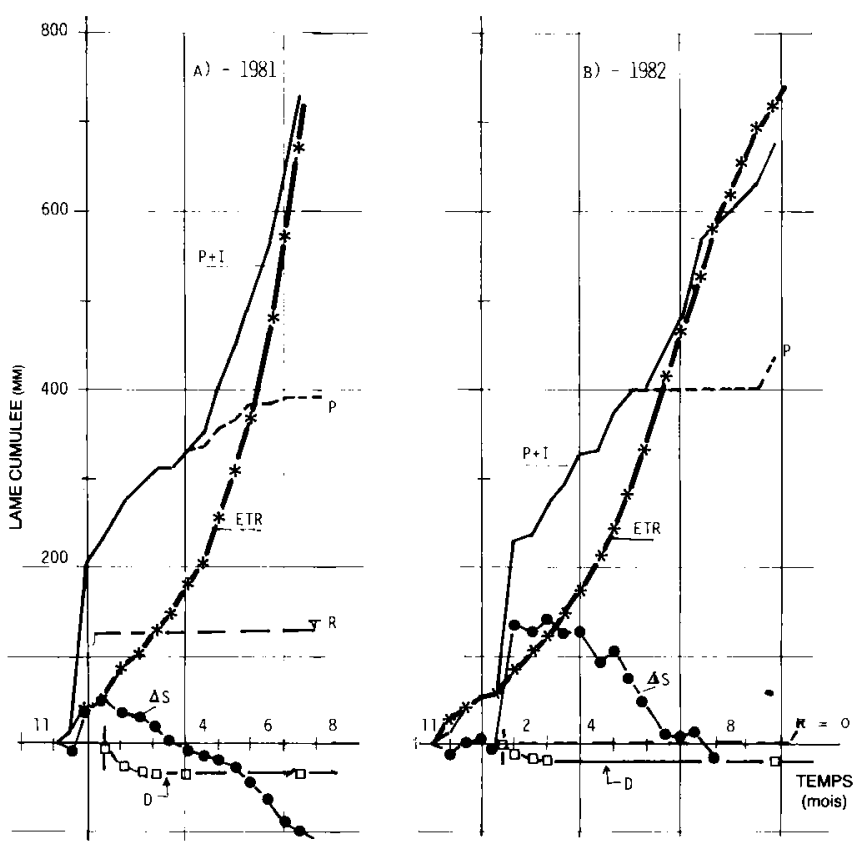

Figure 2

Composantes du bilan hydrique kikuyu (site TK1)

$P$ : pluie ; $I$ : irrigation ; ETR : evapotranspiration réelle

$R$ : ruissellement ; $D:$ drainage ; $\Delta S:$ variation de stock.

Water balance components of kikuyu grass (site TKI)

$P$ : rainfall ; $I$ : irrigation ; ETR : actual evapotranspiration ;

$R:$ runoff $; D:$ drainage ; $\Delta S:$ water storage variation.

Le calcul conduit, d'une part, à un ruissellement de $126 \mathrm{~mm}$ (fig. 2A) en admettant pour la période 1631 décembre $\mathrm{ETR}=\mathrm{ETP}=16 \mathrm{~mm}$ et, d'autre part, à une percolation profonde se manifestant par des variations significatives de la pression effective à $\mathrm{z}=1,75 \mathrm{~m}$ 
TABLEAU 1

Calcul du bilan hydrique sur kikuyu (1 site)

* ruissellement ; ** dose d'irrigation mal répartie.

Calculation of water balance components on kikuyu grass (I site)

* runoff: ** non uniform water application.

\begin{tabular}{crcccc}
\hline \hline Date & \multicolumn{1}{c}{$\mathrm{P}$} & $\mathrm{I}$ & $\Delta \mathrm{S}$ & $\mathrm{D}$ & ETR \\
\cline { 1 - 3 } 01.12 .80 & 15,0 & & $-5,4$ & & \\
16.12 .80 & 187,8 & & $43,6^{*}$ & & 20,5 \\
31.12 .80 & 25,2 & & 10 & 3,6 & 11,6 \\
13.01 .81 & 47,3 & & $-11,6$ & 22 & 37 \\
03.02 .81 & 13,5 & & $-5,9$ & 3,6 & 15,8 \\
18.02 .81 & 22,8 & & $-9,3$ & 2,6 & 29,5 \\
05.03 .81 & 1,0 & & $-17,4$ & 0,6 & 17,8 \\
17.03 .81 & 18,9 & & $-10,8$ & 0,7 & 29 \\
31.03 .81 & 4,5 & 18 & $-6,5$ & 0,3 & 28,7 \\
14.04 .81 & 23,7 & 27 & $-0,9$ & 0,3 & 51,3 \\
28.04 .81 & 8,6 & 36 & $-10,7$ & & 55,3 \\
12.05 .81 & 14,2 & 27 & $-18,4$ & & 59,6 \\
26.05 .81 & & 81 & $-24,3$ & & 105,3 \\
16.06 .81 & 6,9 & $68^{* *}$ & $-20,7$ & & $96,6^{* *}$ \\
30.06 .81 & & 92 & $-5,6$ & & 97,6 \\
14.07 .81 & & & & &
\end{tabular}

( $-5 \mathrm{~m}$ d'eau le 2 janvier $1981 ;-2,7 \mathrm{~m}$ le 6 janvier). Le drainage se prolonge jusqu'au 20 avril. Passé cette date, le gradient hydraulique à $\mathrm{z}=1,60 \mathrm{~m}$ devient nul début juin puis légèrement positif après, traduisant ainsi une tendance aux remontées capillaires. Compte tenu d'un état hydrique plus sec à l'automne 1981 et d'une couverture végétale plus développée par rapport à 1980, aucun ruissellement n'est mesurable lors des fortes pluies de janvier 1982 (fig. 2B). Le drainage induit est plus faible que précédemment et se termine fin mars 1982. Là encore, une tendance à des remontées capillaires se manifeste à partir de juin. Cependant, elles sont quasi négligeables, devant les autres

\begin{tabular}{|c|c|c|c|c|c|}
\hline Date & $\mathbf{P}$ & I & $\Delta \mathrm{S}$ & D & ETR \\
\hline 12.11 .81 & 13,7 & & $-\quad 15,9$ & & 29,6 \\
\hline 01.12 .81 & $\begin{array}{l}13,1 \\
30,9\end{array}$ & & 16,5 & & $\begin{array}{l}2,4,4 \\
14,4\end{array}$ \\
\hline 15.12 .81 & 10,4 & & 2,6 & & 7,8 \\
\hline 29.12 .81 & & & $-\quad 10,1$ & & 10,1 \\
\hline 11.01 .82 & 177,7 & & 153,8 & & 23,9 \\
\hline 02.02 .82 & 8,2 & & $-19,6$ & 10 & 17,8 \\
\hline 17.02 .82 & 35,2 & & 11,1 & 4 & 20,1 \\
\hline 03.03 .82 & 17,2 & & $-\quad 12,7$ & 2 & 27,9 \\
\hline 17.03 .82 & 33,2 & & 6,1 & 2 & 25,1 \\
\hline 30.03 .82 & 1,4 & & $-\quad 39,6$ & & 41,0 \\
\hline 14.04 .82 & 45,7 & & 14,5 & & 31,1 \\
\hline 29.04 .82 & 28,4 & & $-\quad 8,4$ & & 36,8 \\
\hline 12.05 .82 & & & $-50,1$ & & 50,1 \\
\hline 26.05 .82 & & 50 & $-34,5$ & & 84,5 \\
\hline 17.06 .82 & & 30 & $-\quad 22,9$ & & 52,9 \\
\hline 29.06 .82 & & 86,6 & 24,1 & & 62,5 \\
\hline 16.07 .82 & & 16,6 & $-\quad 32,2$ & & 48,8 \\
\hline 29.07 .82 & & $16,6^{* *}$ & $2,9 ?$ & & $?$ \\
\hline 12.08 .82 & & 16,6 & $-\quad 22,1$ & & 38,7 \\
\hline 26.09 .82 & & $16,6^{* *}$ & - $\quad 1,3 ?$ & & $?$ \\
\hline 09.09 .82 & 36 & & 11,2 & & 24,8 \\
\hline
\end{tabular}

termes du bilan, en raison de la faible conductivité hydraulique du sol aux humidités mesurées à $\mathrm{z}=1,60 \mathrm{~m}$.

2 - La non-uniformité des apports d'eau en 1982 sur les périodes 29 juillet- 12 août, d'une part, 26 août09 septembre, d'autre part, rend peu crédible la représentativité spatiale des mesures de variations de stock au site TK1 pour ces 2 périodes.

\section{B. Estimation du bilan hydrique du blé non irrigué}

Comme précédemment, les composantes du bilan hydrique sont données tableau 2, pour les 2 campagnes de mesures.

TABLEAU 2

Calcul du bilan hydrique sur blé : 1 site en 1981, moyenne écart type de 4 sites en 1982

* ruissellement.

Calculation of water balance components on wheat : 1 site in 1981, mean and standard deviation of 4 sites in 1982 * runoff.

\begin{tabular}{crcc}
\hline \hline Date & $\begin{array}{c}\mathrm{P} \\
\mathrm{mm}\end{array}$ & $\begin{array}{c}\Delta \mathrm{S} \\
\mathrm{mm}\end{array}$ & $\begin{array}{c}\mathrm{ETR} \\
\mathrm{mm}\end{array}$ \\
\hline 01.12 .80 & 15,0 & $-1,17$ & 16,7 \\
16.12 .80 & 187,8 & $39,2^{*}$ & $?$ \\
31.12 .80 & 25,2 & 8,4 & 16,8 \\
13.01 .81 & 47,3 & 13,7 & 33 \\
03.02 .81 & 13,5 & 1,2 & 12,3 \\
18.02 .81 & 22,8 & $-7,2$ & 30,0 \\
05.03 .81 & 1,0 & $-21,4$ & 22,4 \\
17.03 .81 & 18,9 & $-15,5$ & 34,4 \\
31.03 .81 & 4,5 & $-20,2$ & 24,7 \\
14.04 .81 & 23,7 & $-5,6$ & 29,3 \\
28.04 .81 & 8,6 & $-6,4$ & 15,0 \\
12.05 .81 & 14,2 & $-6,0$ & 20,2 \\
26.05 .81 & & $-5,3$ & 5,3 \\
16.06 .81 & 6,9 & $-1,9$ & 8,8 \\
30.06 .81 & & -2 & 2 \\
14.07 .81 & & & \\
\hline \hline
\end{tabular}

\begin{tabular}{crrrr}
\hline Date & $\begin{array}{c}\mathrm{P} \\
\mathrm{mm}\end{array}$ & \multicolumn{1}{c}{$\begin{array}{c}\Delta \mathrm{S} \\
\mathrm{mm}\end{array}$} & \multicolumn{2}{c}{$\begin{array}{c}\text { ETR } \\
\mathrm{mm}\end{array}$} \\
\hline 12.11 .81 & 13,7 & $4,5 \pm 2,2$ & $9,2 \pm 2,2$ \\
01.12 .81 & 30,9 & $15,8 \pm 2,9$ & $15,1 \pm 3,9$ \\
15.12 .81 & 10,4 & $-9,8 \pm 1,9$ & $20,2 \pm 1,9$ \\
29.12 .81 & & $-2,4 \pm 0,8$ & $2,4 \pm 0,8$ \\
12.01 .82 & 177,7 & $131,2 \pm 23,6 *$ & $46,5 \pm 23,6$ \\
02.02 .82 & 8,2 & $-24,4 \pm 4,2$ & $32,6 \pm 4,2$ \\
16.02 .82 & 35,2 & $10,5 \pm 3,5$ & $24,6 \pm 3,5$ \\
02.03 .82 & 17,2 & $-19,5 \pm 2,9$ & $36,8 \pm 2,9$ \\
16.03 .82 & 33,2 & $0,1 \pm 2,6$ & $33 \pm \pm 2,6$ \\
29.03 .82 & 1,4 & $-46,1 \pm 6,2$ & $44,7 \pm 6,2$ \\
13.04 .82 & 45,7 & $12,2 \pm 3,1$ & $33,5 \pm 3,1$ \\
27.04 .82 & 28,4 & $-14,5 \pm 5,5$ & $42,9 \pm 5,5$ \\
11.05 .82 & & $-25,6 \pm 2,2$ & $25,6 \pm 2,2$ \\
25.05 .82 & & $-11,0 \pm 3,6$ & $11,0 \pm 3,6$ \\
15.06 .82 & & & &
\end{tabular}


On rappelle que, pour l'année 1981, on ne dispose que du seul site TC1. L'analyse détaillée des profils hydriques et de charge hydraulique correspondants montre que l'essentiel des transferts se produit dans le $1^{\text {er }}$ mètre de sol: aucune variation significative de l'humidité ni de la charge hydraulique (inférieure à $-6,0 \mathrm{~m}$ d'eau) n'a été observée au-delà de la cote $\mathrm{z}=1 \mathrm{~m}$.

Dans ces conditions, on peut légitimement estimer que le drainage est nul. Comme sur gazon, on notera le fort ruissellement consécutif aux pluies de décembre 1980.

En 1982, les bilans ont été effectués sur les 4 sites TC2, TC3, TC4 et TC5 sélectionnés en fonction de leur représentativité spatiale : la figure 3 donne pour chaque site les évolutions temporelles des stocks d'eau contenus entre 0 et $1,75 \mathrm{~m}$, d'une part, entre 1,05 et $1,75 \mathrm{~m}$, d'autre part, et des humidités volumiques à $\mathrm{z}=1,70 \mathrm{~m}$. La figure 4 présente pour chaque site les variations du stock hydrique dans le $1^{\text {er }}$ mètre par rapport au contenu en eau à la date du semis, ainsi que les valeurs de la même variable résultant des prospections gravimétriques à la tarière effectuées en février, avril et

\section{Figure 3}

Variations du stock d'eau entre 0 et $1,75 \mathrm{~m}(-\bullet)$, du stock d'eau entre 1,05 et $1,75 \mathrm{~m}(---\times--)$; de l'humidité à $1,70 \mathrm{~m}(-\Delta-)$ pour les 4 sites sur blé en 1981-82.

Variation in water storage held between 0 and $1.75 \mathrm{~m}(\bullet)$ between 1.05 and $1.75 \mathrm{~m}(---\times--)$ and of volumetric water content at $1.70 \mathrm{~m}(-\Delta-)$ for the 4 sites in or wheat field in 1981-82 period.
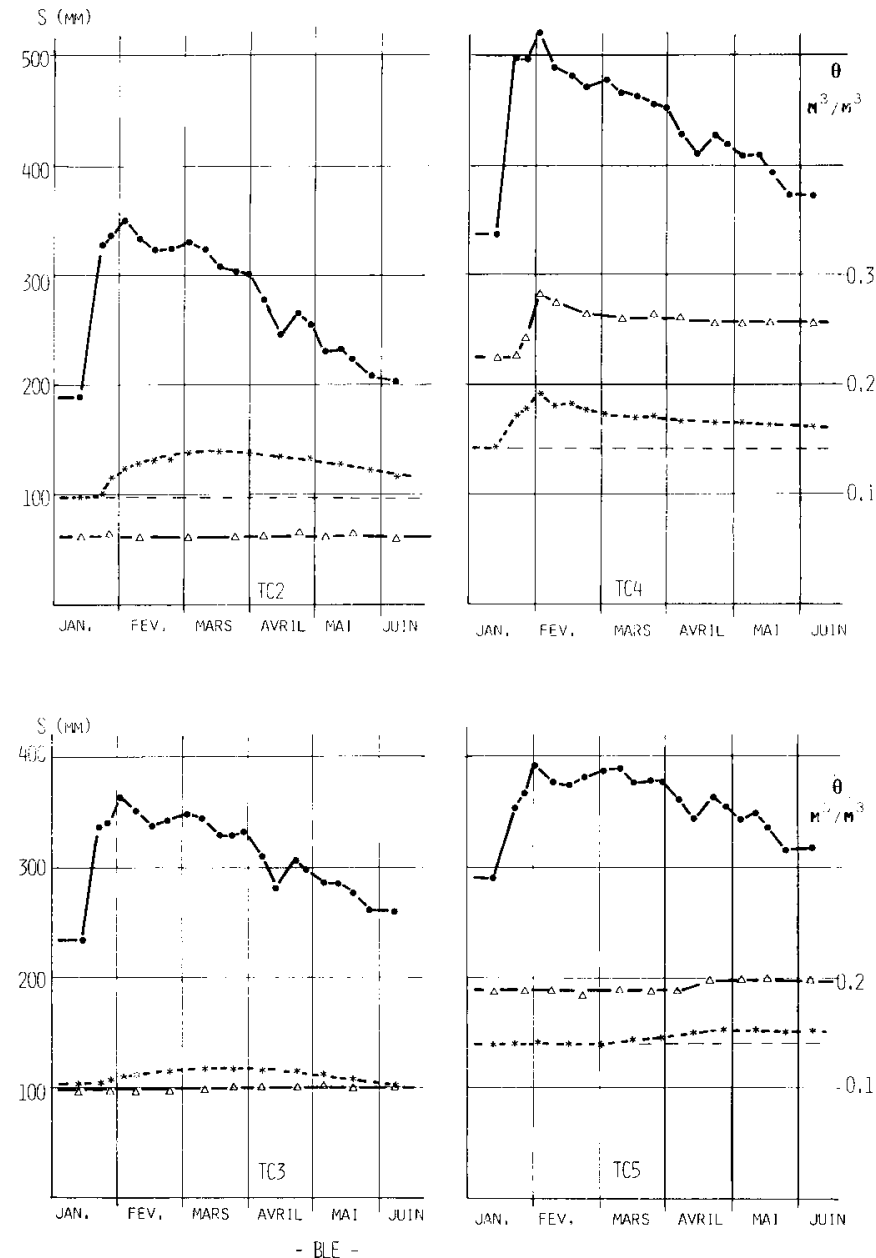

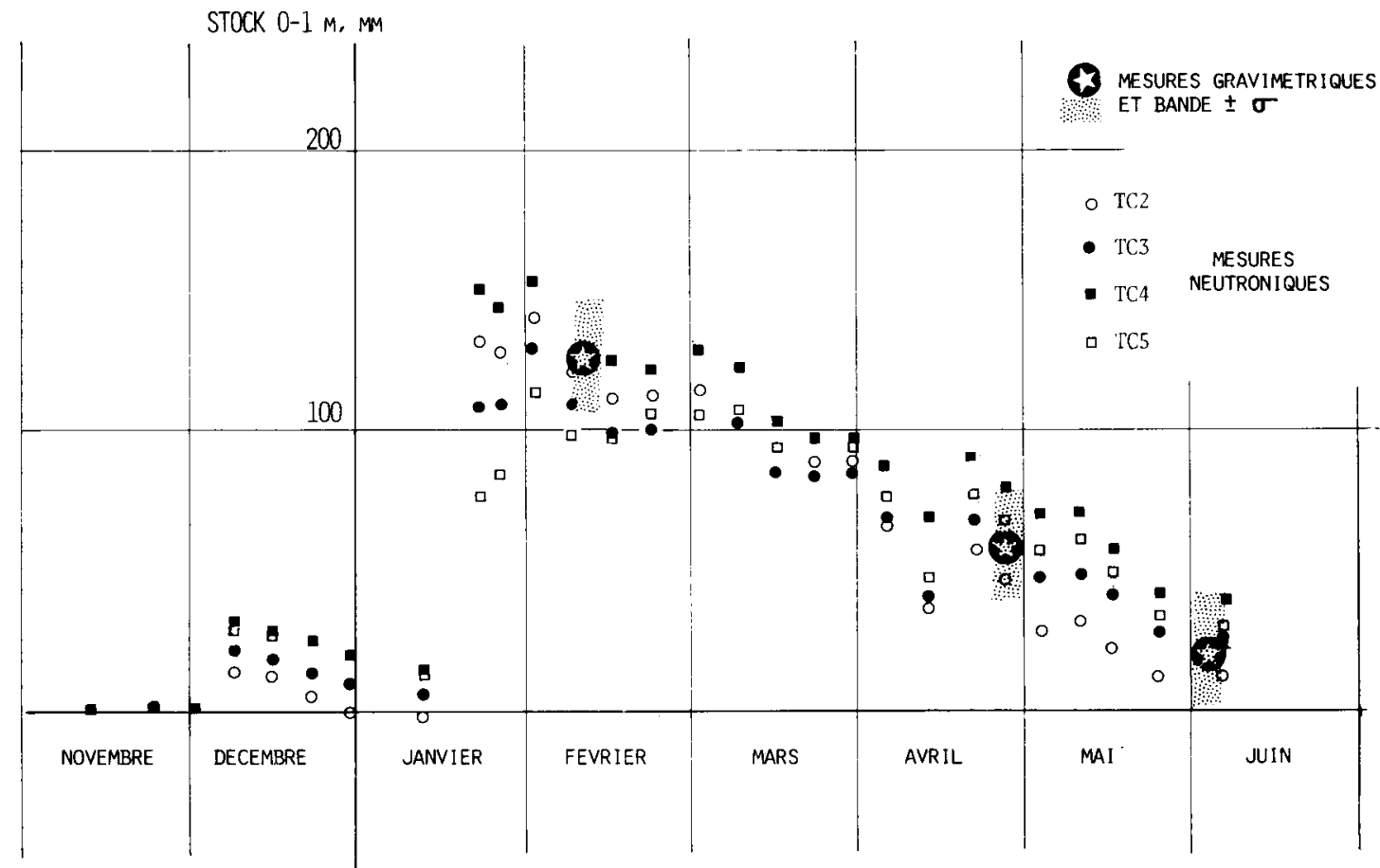

$-1981-$

$-1982-$

$B L E$

Figure 4

Evolution du stock d'eau dans le premier mètre, par rapport à la valeur initiale du 4.11.81, pour les 4 sites sur blé. Comparaison avec les mesures gravimétriques sur toute la parcelle.
Change with time in water storage held in the first meter, normalized for the initial value measured on 4.11.81 for the 4 sites on the wheat field. Comparison with gravimetric measurements over the entire field. 
juin. L'analyse de ces 2 figures suscite les remarques suivantes :

1 - L'essentiel des variations du stock hydrique a lieu dans le $1^{\text {er }} \mathrm{m}$ (fig. 3) à l'exception peut-être du site TC4. Sur ce dernier, beaucoup plus argileux que les autres, on peut observer une augmentation importante du stock d'eau en dessous de $1 \mathrm{~m}$ et une humidification significative de la teneur en eau à $z=1,70 \mathrm{~m}$ après les pluies de janvier 1982. Le calcul du drainage conduit à une lame d'eau écoulée de $25 \mathrm{~mm}$ entre le 26 janvier et le 6 avril.

Cependant, l'étude de la distribution spatiale des fronts de pénétration de la pluie (VACHAUD et al., 1983) a montré que la probabilité d'occurrence de drainage à cette cote ne représente pas plus de $5 \mathrm{p} .100$ de la surface totale de la parcelle. Cela correspond à une lame moyenne écoulée de $1,25 \mathrm{~mm}$ qui peut être négligée dans le calcul du bilan moyen donné tableau 2.

2 - Il existe de grandes différences entre les valeurs absolues du stock hydrique de site à site: près de $100 \mathrm{~mm}$ d'écart entre les valeurs extrêmes (fïg. 3). En revanche les variations relatives sont plus voisines (fig. 4). Cela met clairement en évidence le rôle d'homogénéisation joué par la végétation vis-à-vis de la consommation en eau.

3 - La procédure adoptée pour le choix des sites de mesure est justifiée par les résultats de la figure 4 . En effet, les valeurs obtenues sur les $\mathbf{4}$ sites sont situées à l'intérieur de la bande de certitude entourant la moyenne calculée à partir des prélèvements gravimétriques quadrillant l'ensemble de la parcelle. Le site TC3 reste voisin du site moyen, TC4 représente l'extrême humide et TC2 l'extrême sec.

La figure 5 présente les valeurs cumulées des différentes composantes du bilan hydrique. Les résultats relatifs à l'année 1981 n'ont qu'une valeur symbolique, puisque obtenus sur le seul site TC1. L'année suivante, les courbes ETR et $\Delta S$ correspondent aux moyennes spatiales associées à leur bande confiance, dont les valeurs numériques sont données tableau 2 . On notera que durant les périodes pluvieuses, le bilan a été effectué en posant ETR = ETP. La forte variance observée sur la période 12 janvier-02 février 1982 est probablement imputable à la variabilité locale des sites de mesures (piétinement, effets micro-topographiques) qui influence l'infiltrabilité du sol, donc le ruissellement induit.

\section{DISCUSSION : COMPARAISON ETR/ETP}

Les valeurs des flux journaliers d'évapotranspiration réelle (ETR) calculées par humidimétrie neutronique et tensiométrie, et potentielle (ETP) estimées par la formule de PENMAN, (RIOU \& CHARTIER, 1985) sont présentées figure 6 pour le gazon et le blé.

On notera que les valeurs décadaires de l'ETP données par RIOU \& CHARTIER (1985), ont été recalculées ici pour correspondre aux dates des mesures hydriques. De plus, les valeurs ETP-blé ne représentent la consommation maximale que pour un blé bien développé (avril-mai). En effet, ces valeurs ont été calculées par ces auteurs en prenant une longueur de rugosité aérodynamique constante et égale à celle relative à la culture en fin de croissance.

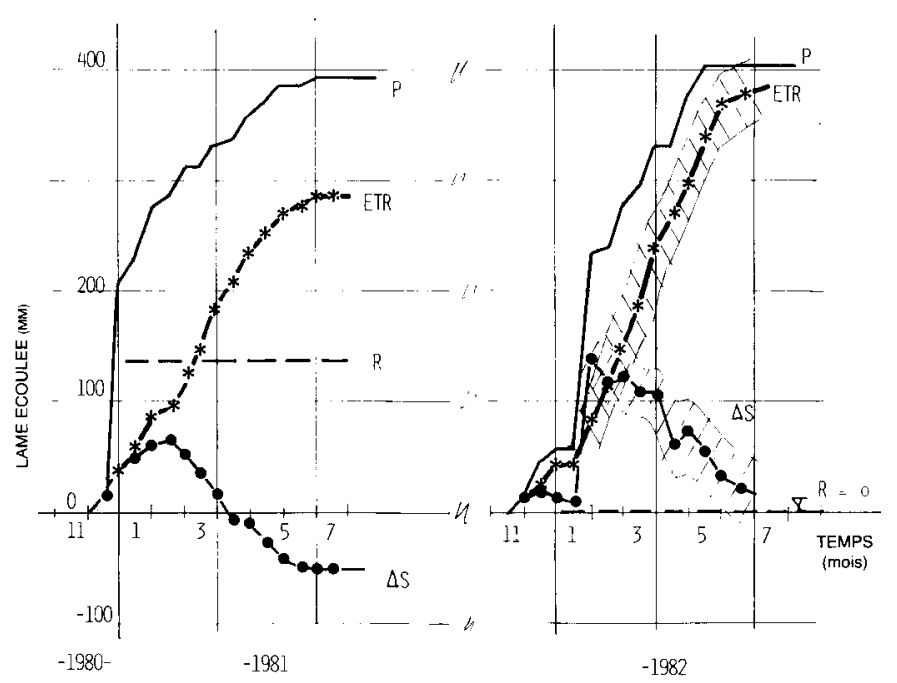

Figure 5

Composantes du bilan hydrique sur blé: site TCI en 1981; moyenne de 4 sites en 1982. Les bandes hachurées correspondent aux domaines de certitude des valeurs moyennes à $68 \mathrm{p} .100 . P$ : pluie ; $R$ : ruissellement; ETR : évapotranspiration réelle; $\Delta S$ : variation de stock.

Water balance components of wheat : site TC1 in 1981 ; average of 4 sites in 1982. The hatched areas represent the confidence intervals at $68 \%$ level. $P$ : rainfall ; $R$ : runoff ; ETR : actual evapotranspiration; $\Delta S:$ water storage variation.

\section{A. Cas du gazon}

Globalement sur l'ensemble des 2 années d'étude, on constate un bon accord entre les valeurs d'ETR et d'ETP tant que les apports d'eau sont suffisants. Cependant en raison d'une répartition pluviométrique différente entre les 2 années, la période pendant laquelle l'eau dans le sol n'est pas limitante se termine fin février 1981 mais s'étale l'année suivante jusqu'au 15 mai. En effet, en 1981 la faible pluviométrie associée à une irrigation insuffisante durant la période $1^{\text {er }}$ mars-14 avril (tabl. 1) est responsable de la chute de l'ETR par rapport à ETP. Au-delà, les apports d'irrigation permettent de maintenir le gazon en évapotranspiration potentielle, sauf à 2 périodes : 12-26 mai, 16-30 juin. On notera que sur la période allant du $1^{\text {er }}$ janvier au 14 juillet, l'ETR totale mesurée $(634 \mathrm{~mm})$ est très voisine de l'ETP $(660 \mathrm{~mm})$.

En revanche, en 1982, on constate dès le début de l'irrigation (9 mai) une différence sensible et croissante entre ETR gazon et ETP. A l'évidence, le sol n'est pas à même de fournir les compléments d'eau aux doses d'irrigations qui, compte tenu de la forte demande évaporative ont été notoirement insuffisantes.

Les données manquantes sur les valeurs d'ETR gazon correspondent à des valeurs incorrectes, résultant de l'irrégularité de la répartition des apports d'irrigation (cf. tabl. 1).

\section{B. Cas du blé}

Il paraît intéressant de noter les points suivants :

1 - Les résultats obtenus sur 2 années consécutives montrent qu'en début de cycle (janvier-février) les 


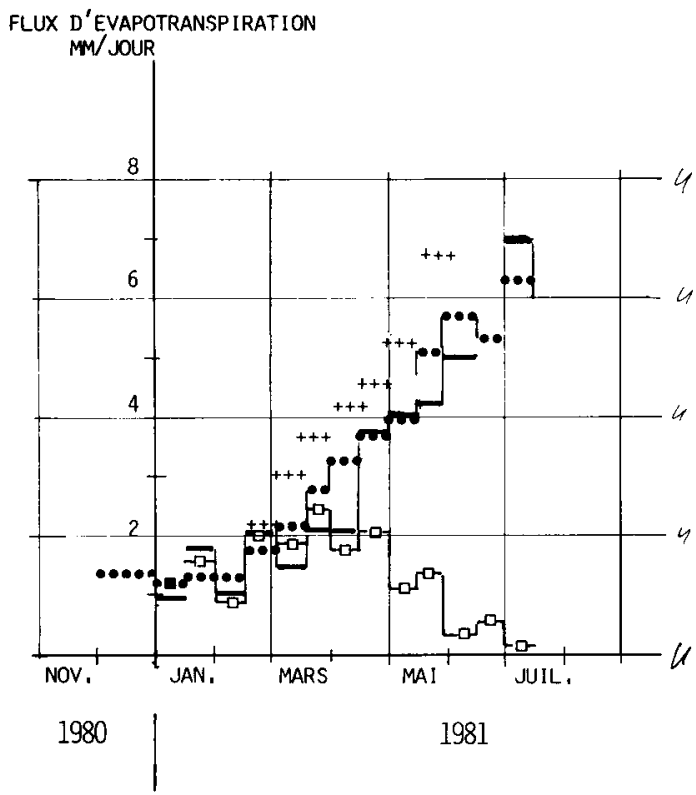

Figure 6

Comparaison entre les mesures de flux moyen journalier d'ETR sur ble ( - - - et kikuyu (—) et les estimations d'ETP sur blé $(+++)$ et

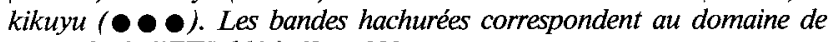
certitude de l'ETR blé à 68 p. 100.

valeurs d'évapotranspiration réelle sont très voisines de celles mesurées sur gazon, elles-mêmes peu différentes des valeurs potentielles. Il apparaît donc justifié d'estimer en hiver l'ETR-blé par l'ETP-gazon. Cela paraît intéressant dans la pratique dans la mesure où la détermination de la consommation par les mesures dans le sol pose problème en cas de ruissellement consécutif à des pluies intenses et non rares en zones semi-arides.

2 - De mi-février à fin mai, l'ETP-blé est systématiquement supérieure à celle du gazon. RIOU \& CHARTIER (1985) attribuent ce comportement au fait que la fonction vent, intervenant dans la formule de PENMAN sur blé en phase de croissance, est nettement supérieure à celle du gazon.

3 - En fin de cycle, on notera la décroissance très brutale de l'ETR en relation avec la diminution, d'une part, des besoins physiologiques en eau et, d'autre part, de la réserve hydrique du sol. Faute de mesures sur le végétal, il n'a pas été possible de déterminer le facteur le plus explicatif.

\section{CONCLUSIONS}

Au terme de cette étude, il paraît intéressant d'insister sur la complémentarité des 2 approches présentées.

Dans la $1^{\text {re }}$ partie de cet article, RIOU \& CHARTIER montrent l'intérêt des méthodes d'estimation de l'ETP-gazon, soit par corrélation avec des mesures simples (bac), soit par utilisation de la formule de PENMAN, préalablement ajustée aux conditions locales.

Dans cette $2^{e}$ partie, il apparaît que si l'on prend soin de choisir des sites de mesures représentatifs de la parcelle, les déterminations d'évapotranspiration des 2 couverts considérés par la méthode du bilan hydrique sont tout à fait compatibles avec les estimations d'ETP dans le cas où l'eau dans le sol n'est pas le facteur limitant. Lorsque les conditions d'alimentation hydrique deviennent défavorables, cette méthode permet de

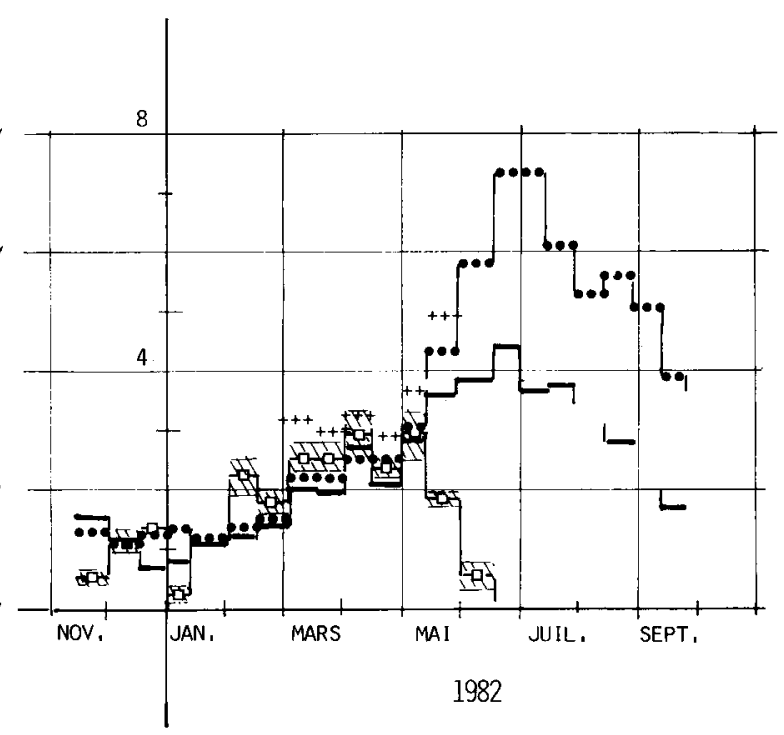

Comparison between the measured actual evapotranspiration daily rates on wheat ( $-\square-$ ) and kikuyu grass (-) and the estimated potential daily rates on wheat $(+++)$ and kikuyu grass $(\bullet \bullet \bullet)$. The hatched areas represent the confidence intervals at $68 \%$ level for actual evapotranspiration rates of wheat.

quantifier l'évapotranspiration réelle, donc les déficits hydriques.

Les résultats mettent en évidence le problème lié à l'estimation par la méthode du bilan hydrique de l'évapotranspiration en cas de ruissellement, puisqu'il s'agit de déterminer 2 inconnues (ETR et R) avec une seule équation. Le couplage de cette méthode avec une estimation de l'ETP (où l'évapotranspiration maximum du couvert) devrait permettre alors de lever cette ambiguïté en utilisant l'algorithme simple suivant :

- estimation de l'ETR en supposant l'absence de ruissellement :

$$
\mathrm{ETR}=\mathrm{P}+\mathrm{I}-\mathrm{D}-\Delta \mathrm{S}
$$

- comparaison ETR/ETP :

si ETR obtenu par (4) $>$ ETP alors on prend ETR $=$ ETP et $\mathrm{R}=\mathrm{P}+\mathrm{I}-\mathrm{ETP}-\mathrm{D}-\Delta \mathrm{S}$

si ETR < ETP alors R = 0 et ETR est donnée par (4)

L'expérimentation présentait 2 lacunes : l'absence de mesures physiques et physiologiques sur les couverts végétaux confère à cette étude un aspect plus méthodologique qu'agronomique ; le caractère trop épisodique des mesures du bilan radiatif, des profils de vent et de température au-dessus de la végétation n'a pas permis la mise en œuvre de la méthode aérodynamique pour estimer l'évapotranspiration réelle, en cas d'alimentation hydrique limitante. Ces aspects doivent faire l'objet d'une étude future sur le même site, afin d'effectuer une comparaison plus complète entre les 2 approches expérimentales.

Reçu le 7 février 1984. Accepté le 5 novembre 1984.

\section{REMERCIEMENTS}

Cette étude a été faite dans le cadre d'une convention de recherches entre le Centre National de la Recherche Scientifique (CNRS) Paris et la Direction de la Recherche Scientifique et Technique (DRST) Tunis.

Les auteurs tiennent à remercier les collaborateurs ayant participé aux campagnes de mesure pour le travail effectué. 


\section{RÉFÉRENCES BIBLIOGRAPHIQUES}

Dancette C., Hamon G., Vachaud G., 1979. Etude comparée de la dynamique de l'eau en sol sableux et cultivé. In: "Isotopes and Radiations in Research on soil-plant relationships ". Int. Symp., Colombo, ed. par AIEA, Vienne, 213-214.

Haverkamp R., Vauclin M., Vachaud G., 1984. Error analysis in estimating soil water content from neutron probe measurements. 1. - Local standpoint, Soil Sci., 137, 78-90. II. - Spatial standpoint, Soil Sci., 137, 141-148.

Kalms J. M., Vachaud G., Vauclin M., 1982. Modalités d'alimentation hydrique de deux variétés de riz pluvial à l'échelle d'une parcelle avec prise en compte de la variabilité spatiale du sol. Agronomie, 2 (9), 871-883.

Perrier A., Katerji N., Gosse G., Itier B., 1980. Etude in situ de l'évaporation réelle d'une culture de blé. Agri. Météor., 21, 295-311.
Riou C., Chartier R., 1985. Evapotranspiration en zone semi-aride des couverts végétaux (gazon, blé) obtenue par plusieurs méthodes. 1. - Evaluation de I'ETP (conditions hydriques non limitantes), Agronomie, 5 (3), 261-266.

Vachaud G.. Chaabouni Z.. El Amani S., Vauclin M.. 1983. Méthodologie d'etude du bilan hydrique d'une culture à l'échelle d'une parcelle. In : "Emploi des Techniques isotopiques et des Rayonnements dans les Etudes sur la physique des sols et l'Irrigation ». Coll. int., Aix-en-Provence, IAEA ed., Vienne, 79-101.

Vachaud G., Passerat A., Balabanis P., Vauclin M., 1984. Temnoral stability of spatially measured soil water probability function. Soil Sci. Soc. Am. J. (accepte pour publication). 